\title{
REG $\gamma$ is associated with multiple oncogenic pathways in human cancers
}

\author{
Jing He ${ }^{1,4 \dagger}$, Long Cui ${ }^{2^{*}+}$, Yu Zeng ${ }^{1}$, Guangqiang Wang ${ }^{1}$, Ping Zhou ${ }^{1}$, Yuanyuan Yang ${ }^{1}$, Lei $\mathrm{Ji}^{1}$, Yanyan Zhao ${ }^{1}$, \\ Jiwu Chen ${ }^{1}$, Zhuo Wang ${ }^{1}$, Tieliu Shi ${ }^{1}$, Pei Zhang ${ }^{3}$, Rui Chen ${ }^{4}$ and Xiaotao Li $i^{1,4^{*}}$
}

\begin{abstract}
Background: Recent studies suggest a role of the proteasome activator, REG $\gamma$, in cancer progression. Since there are limited numbers of known REG $\gamma$ targets, it is not known which cancers and pathways are associated with REG $\gamma$.

Methods: REG $\gamma$ protein expressions in four different cancers were investigated by immunohistochemistry (IHC) analysis. Following NCBI Gene Expression Omnibus (GEO) database search, microarray platform validation, differential expressions of REG $\gamma$ in corresponding cancers were statistically analyzed. Genes highly correlated with REG $\gamma$ were defined based on Pearson's correlation coefficient. Functional links were estimated by Ingenuity Core analysis. Finally, validation was performed by RT-PCR analysis in established cancer cell lines and IHC in human colon cancer tissues
\end{abstract}

Results: Here, we demonstrate overexpression of REG $\gamma$ in four different cancer types by micro-tissue array analysis. Using meta-analysis of publicly available microarray databases and biological studies, we verified elevated REG $\gamma$ gene expression in the four types of cancers and identified genes significantly correlated with REG $\gamma$ expression, including genes in p53, Myc pathways, and multiple other cancer-related pathways. The predicted correlations were largely consistent with quantitative RT-PCR analysis.

Conclusions: This study provides us novel insights in REG $\gamma$ gene expression profiles and its link to multiple cancerrelated pathways in cancers. Our results indicate potentially important pathogenic roles of REG $\gamma$ in multiple cancer types and implicate REG $\gamma$ as a putative cancer marker.

\section{Background}

REG $\gamma$, also known as PA28gamma, 11Sgamma, or PSME3, was first identified as Ki antigen, a nuclear protein targeted by autoantibodies found in sera of patients with systemic lupus erythematosus [1]. It is a member of the 11S family of proteasomal activators that have the ability to stimulate the proteolytic activity of the $20 \mathrm{~S}$ core proteasome independent of ubiquitination and ATP [1]. Accumulating evidence suggests REG $\gamma$ is involved in cancer progression [2]. REG $\gamma$ has been reported to be overexpressed in colorectal cancer [3] and thyroid cancer [2], and is involved in cancer development $[2,4-6]$. It is unknown, however, whether

\footnotetext{
* Correspondence: longcuidr@yahoo.com.cn; xiaotaol@bcm.edu

+ Contributed equally

${ }^{1}$ Institute of Biomedical Sciences, East China Normal University, 500

Dongchuan Rd., Shanghai 200241, China

2Department of Colorectal Surgery, Xin-hua Hospital, Shanghai Jiao-tong

Univerisy, Shanghai, People's Republic of China

Full list of author information is available at the end of the article
}

REG $\gamma$ is involved in additional cancers. REG $\gamma$ is known to degrade both oncogenic and tumor suppressing proteins such as SRC-3, HCV core protein, PTTG1, p21, p16, p19, and $\mathrm{p} 53$. In this study we try to understand expression profiles of REG $\gamma$ in multiple cancer types and correlations of REG $\gamma$ with known cancer or cancer related pathways.

Microarray assays have been widely adopted in cancer marker exploration and expression profiling of tumor genes $[3,4]$. Microarray studies have contributed valuable information to our understanding of cancer by identifying biomarkers and enabling classification of tumor subtypes [5-8].

In this study, we focused on thyroid cancer, colon cancer, liver cancer and lung cancer since the first two cancers were reported with over-expression of REG $\gamma[3,9]$ and the other two are among the list of the most malicious cancers. We analyzed REG $\gamma$ expression in cancer tissue arrays by using publicly available microarray data from NCBI GEO database. We acquired datasets and integrated

\section{() Biomed Central}


the analyzed results across different datasets and cancer types to characterize a general REG $\gamma$ expression pattern in four different cancer types by comparing human cancer versus normal tissues. We set clear criteria along with quality controls for dataset screening and normalization, which allowed us to carry out comprehensive datasetbased meta-analysis across differing cancers. A set of genes highly correlated with REG $\gamma$ expression were identified and validated by RT-PCR to identify putative functional interactions associated with REG $\gamma$.

\section{Methods}

\section{Cell types and cell culture}

A549, HepG2, and HCT116 cells were purchased from ATCC and maintained at Cell Culture Core at the Department of Cell Biology, BCM. The human thyroid carcinoma cell line ARO was kindly provided by Dr. Adel El-Naggar at the University of Texas M.D. Anderson Cancer Center. The ARO cell line was authenticated at Genotyping Center of John's Hopkins University. The shN and shR stable cell lines were generated in ARO, A549, and HCT116 by introducing retroviral shRNA vectors specific for REG $\gamma$ or a control vector from OriGene (Rockville, MD). ARO cells were cultured in 1640 supplemented with $10 \%$ fetal bovine growth serum (GIBCO). All other cells were cultured under standard conditions described by the ATCC.

\section{Immunohistochemical assay}

IHC analysis was performed to analyze REG $\gamma$ expression of protein level in several human cancers including lung, colon, thyroid and liver cancer. Sections were deparaffinised and rehydrated. The slides were then heated in a $100^{\circ} \mathrm{C}$ water bath for 30 minutes in a $0.01 \mathrm{M}$ citrate buffer solution at $\mathrm{pH}$ 6.0, and cooled to room temperature. After quenching the endogenous peroxidase activity with $0.3 \%$ $\mathrm{H} 2 \mathrm{O} 2$ (in absolute methanol) for 10 minutes, the sections were treated for 10 minutes at room temperature with the serum albumin (HISTOSTAIN-PLUS DAB kit) to block non-specific staining. Duplicate sections were incubated overnight in $4^{\circ} \mathrm{C}$ with the primary specific antibodies. Slides were then incubated for 10 minutes with biotinylated anti-rab-bit IgG (DAB kit) for REG $\gamma$ recognition. The sections were incubated with the HRP for 10 minutes. Finally, the sections were counterstained with Mayer's haematoxylin.

\section{Preliminary datasets collection}

Microarray expression profiles were obtained from Gene Expression Ominibus of National Center of Biotechnology Institute [10]. All datasets in this study were published within the past 5 years (2004-2009) and following the Minimum Information about a Microarray Experiment (MIAME) guidelines, including 49 datasets, with 3,832 samples containing 16, 15, 11, and 7 datasets from colon, liver, lung and thyroid cancer, respectively (Additional file 1: Table S2). The following preliminary datasets were retrieved: a) primary tumors, carcinoma and adenoma along with normal controls in each tissue: primary colon cancer samples including early onset colorectal carcinoma, colon tumor and adenoma; primary hepatocelluar carcinoma (HCC); lung cancer including non-small cell lung cancer, adenocarcinoma, and squamous cell carcinoma; thyroid cancer samples including papillary thyroid carcinomas (PTC), anaplastic thyroid carcinoma (ATC), follicular carcinomas (FC) and follicular adenomas. b) noncancer diseases originated from colon, liver, lung and thyroid tissues, including inflammatory bowel disease (IBD), Crohn's disease (CD), ulcerative colitis (UC), HCV cirrhosis, HCV-induced dysplasia, pneumonia, and follicular goiter. c) different stages of cancers with a stage 0 tissue or healthy or distant adjacent tissues as control. The following datasets/samples were excluded: 1) datasets with no contorl tissue; 2) datasets without REG $\gamma$ probe/probe set included in platform; 3) datasets without corresponding publication; 4) datasets with samples collected in time courses; 5) datasets without gene symbol annotation for the probes by the Human Gene Nomenclature (HGNC) guidlines; 6) datasets without REGy data in the microarray platform. The preliminary sample screening yielded 23 datasets $(n=1,070)$ for differentially expressed gene analysis (Additional file 2: Table S3). Of these output, there were 15 cancer datasets $(n=614), 2$ non-cancer diseases datasets of $(n=86)$, and 6 datasets ( $\mathrm{n}=399)$ containing both cancer $(\mathrm{n}=260)$ and disease samples $(\mathrm{n}=139)$.

\section{Dataset based expression analysis}

Microarray datasets (described above) were analyzed by GEOquery and Limma packages in $\mathrm{R}$ http://www.r-project.org/ as described previously [11-13]. First, all raw data were downloaded from GEO and mono-channel data were normalized using MAS5.0. Samples in each dataset were grouped into three classes, namely cancer, non-cancer disease and normal samples. The $\log 2$ ratio values of disease group versus normal group were calculated based on the normalized data. For all two-channel datasets, $\log 2$ transformed expression ratios were calculated and used in all subsequent analyses. Two-sample paired t-test [14] were carried out between cancer vs. non-cancer diseases and cancer vs. normal following statistic analysis as described $[15,16]$. Internal quality controls were set up for each dataset by validating the statistical significance of specific genes with what was reported in relevant publications. Two-sample comparisons were statistically analyzed for all 21 cancer datasets containing 874 cancer samples and 625 paired normal samples. An additional two-sample comparison was performed with the 8 non-cancer disease 
datasets, including 196 non-cancer disease samples and 174 normal control samples (Additional file 2: Table S3).

\section{Correlation analysis}

The Pearson's Correlation Coefficient (PCC) was used as a measure of correlation between REG $\gamma$ and its potentially related genes based on 13 datasets, 4 from liver $(n=299)$ and 3 from each of lung $(n=164)$, colon $(n=77)$, and thyroid $(n=126)$ respectively. Pearson Correlation analysis was conducted using $\mathrm{R}[15,17]$ on datasets with significant overexpression of REG $\gamma$. PCC of REG $\gamma$ with each gene in each dataset was calculated. Genes whose expression correlated with REG $\gamma$ in each dataset were ranked based on their p-value. In order to produce at least 600 candidates in each datasets for subsequently selection, we used a cutoff of 0.001 . The top $20 \%, 15 \%, 10 \%$, and $5 \%$ genes were selected from thyroid, colon, liver, and, lung cancer datasets respectively. All subsequent selections and analyses were based on these genes referred to as REG $\gamma$ correlated genes.

Genes were selected from all REG $\gamma$ correlated genes based on cancer type except for the initial pilot testing (a PCC cutoff as \pm 0.6 [18]). Our criteria were that each gene was present in at least 2 datasets, according to binomial distribution $(p<0.05)$, in one cancer type and the cutoff of PCC in one cancer type was set to \pm 0.6 . Genes that fulfill these criteria were considered as highly-correlated with REG $\gamma$ and used for downstream pathway analysis.

\section{Pathway and network analysis}

Genes highly correlated with REG $\gamma$ were analyzed with the IPA (Ingenuity Pathway Analysis) system: http:// www.ingenuity.com. With core analysis, all qRT-PCR validated $R E G \gamma$-correlated genes were mapped and then analyzed using Ingenuity Knowledge Base (genes only) to yield bio-function pathway annotation and networks showing direct and indirect relationships between genes and molecules.

To calculate the composition of REG $\gamma$-correlated genes/pathways in cancers, results from Ingenuity pathway analysis were grouped into three clusters: cancer pathways, cancer related pathways, and other pathways. These pathway clusters were grouped based on the following characterization: 1) cancer pathways included bio-function of cancer, tumor or tumorigenesis, neoplasia, carcinoma or adenocarcinoma, lymphoma and sarcoma; 2) cancer related pathways included a) pathways related to cell cycle with following bio-function category: mitosis or mitotic, G2/M/S phase, cell division, check point, and arresting; b) cell growth pathways involved in: survival, growth and proliferation; c) cell death pathways with bio-function of apoptosis and death. 3) Other pathways: all the rest of the pathways not included in cancer or cancer-related pathway clusters.
Genes highly correlated with REG $\gamma$ were also searched against the KEGG pathways database http://www.genome.jp/kegg/tool/colorpathway.html to highlight and augment the published graphical pathways analyzed by Ingenuity. Protein-protein interaction network analysis was performed by checking REG $\gamma$ highly-correlated genes in the STRING database http://string-db.org) [19]. To make the network concise, genes with connections equal or greater than 3 were selected.

\section{PCR validation}

Confirmatory qRT-PCR was performed on randomly selected REG $\gamma$ correlated genes. Fifteen genes were selected from the REG $\gamma$-correlated genes and an additional fifteen genes highly correlated with REG $\gamma$ expression were selected for qRT-PCR. RT-PCR experiments were carried out in cells originated from colon, liver, lung and thyroid cancer.

\section{RNA preparation, qRT-PCR and RNAi}

Cells were grown to $75 \%$ confluence in a $6 \mathrm{~cm}$ dish and lysed with buffer provided in RNA extraction kit (TAKARA) and RNA was extracted following the manufacturer's instruction. RNA quality and integrity were verified by gel electrophoresis. Two-microgram of total RNA was reverse transcribed with M-MLV reverse transcriptase (Invitrogen). Gene-specific primers were designed as follow: REG $\gamma$ forward primer, 5'-TCCTCACCAATAGCCACG-3'; REG $\gamma$ reverse primer, 5'-CTCGATCAGCAG CCGAAT-3'; 18S rRNA forward primer, GGACACGGACAGGATTGACA; 18S rRNA reverse primer, GACATCTAAGGGCATCACAG. qRT-PCR was conducted using the SYBR ${ }^{\circledR}$ PrimeScriptTM RT-PCR Kit (TAKARA). The results were analyzed using the MxPro qRT-PCR software.

RNA interference was performed in HepG2 cells to knock down REG. HepG2 was seeded in 6-well plate at $60 \%$ confluence overnight and was transfected with 10 nM siRNA along with lipofection 2000. Cells were harvested 72 hours later for RNA extraction and qRT-PCR analysis.

\section{Statistic analysis}

Weighted student t-test for two-sample with unequal variance was used to calculate statistics and p values for IHC tissue array [16]. Two-tailed student's t-test was used in microarray expression analysis and Fisher's $\mathrm{Z}$ transformation was used to adjust $\mathrm{p}$ value. A p-value of less than 0.05 was defined as "significant" for all statistic analysis involved in expression analysis. Datasets in which REG $\gamma$ is highly significant $(p<0.001)[18]$ were selected for subsequent correlation analysis. In correlation analysis, Pearson's correlation coefficient was set with a cutoff PCC \pm 0.6 [18] and binomial coefficient was used based on datasets number in each cancer type to selection REG $\gamma$ highly 
related genes. Pathways with a p-value less than 0.01 were chosen to be studied in Ingenuity core analysis.

\section{Results \\ REG $\gamma$ protein is highly expressed in multiple cancers}

To understand whether REG $\gamma$ is a tumor-associated protein, we examined REG $\gamma$ expression levels in multiple human carcinomas. IHC experiment was performed using tissue arrays containing 92 cases of primary lung cancer, 48 colon cancers, 49 thyroid cancers, and 206 liver cancer samples along with corresponding normal tissues, all arranged in duplicates (Table 1, Additional file 3: Table S1). The expression of REG $\gamma$ in cancer samples was scored double-blindly by comparing with normal tissues or adjacent non-cancer tissues which have no positive staining or low levels of REG $\gamma$ staining (Figure 1 and Table 1). The scored REG $\gamma$ expression is consistent for most of the duplicate samples and a representative scored result was shown in Additional file 3: Table S1. The overall rate of REG $\gamma$ overexpression (a combination of ++ and +++ staining) in different carcinoma is higher than $50 \%$. We observed a statistically significant increase in the number of late-stage cancers with the highest REG $\gamma$ expression $(+++)$, such as in stage III of adenocarcinoma and squamous cell carcinoma (Additional file 3: Table S1). Our results provide the first evidence for an association of REG $\gamma$ with primary human lung carcinoma and liver cancer, substantiating previous observations that $\mathrm{REG} \gamma$ is increased in colon and thyroid cancers.

\section{Integrated analysis of microarray datasets revealed overexpression of REG $\gamma$ in selective cancers}

Overexpression of REG $\gamma$ protein in four different human cancers prompted us to investigate whether elevation of REG $\gamma$ is regulated at the mRNA level. We searched GEO database by keywords and identified 49 datasets (Additional file 1: Table S2), of which 23 were qualified for expression analysis in this study (Figure 2A). Significantly higher REG $\gamma$ expression ( $\mathrm{p}<0.05$ ) was observed in $67 \%$ of cancer datasets (14 of $21, \mathrm{n}=597$ ) when compared with normal tissues (Figure 2B). Consistently, our comparative analysis of control vs. non-cancer diseases [20-22], revealed that most of the non-cancer datasets had no significant differences in REG $\gamma$ expression (66\%, $n$ $=117)$. On the contrary, only small percent of cancer datasets ( 7 of $21, \mathrm{n}=228$ ) had no significant elevation in REG $\gamma$ levels (Figure 2B), indicating potential association of REG $\gamma$ in the development of these cancers. Cancer type based analysis indicated an increase of REG $\gamma$ in 60$83 \%$ of cancer datasets (Figure $2 \mathrm{C}$ ), concordant with our IHC studies (Figure 1).

A detailed analyses of pathologically classified, stagespecific cancers and non-cancer diseases were executed using dataset GSE6764 [23], GSE4183 [22], GSE6339 [21] and GSE7670 [20], which originate from liver, colon, thyroid, and lung respectively and disclosed detailed cancer stage information (Additional file 2: Table S3). The representative REG $\gamma$ expression patterns in the four cancers, non-cancer diseases, normal controls, and some staged cancer samples are illustrated in Figure 3. Statistically significant elevation of REG $\gamma$ gene expression in cancers ranged from 1.25 to 2.43 fold-change (overexpression cut-off: fold change $>1$ ), consistent with our IHC result in corresponding cancer tissue arrays (Figure 1). In liver cancer samples, REG $\gamma$ appeared considerable up-regulation, consistent with the original publication where potential cancer biomarkers were linked with stepwise carcinogenic process [23]. The results of stage-associated increases of REG $\gamma$ in advanced liver cancers in Figure 3A is consistent with our observation of higher REG $\gamma$ staining in advanced cancer types. For most of the non-cancer datasets, the p-values of disease classes, such as IBD \& CD, showed no significant changes in REG $\gamma$ expression. In conclusion, expression of REG $\gamma$ is significantly increased in multiple human cancer types and likely involved in late-stage cancers.

Table 1 Summary of IHC analysis of REG $\gamma$ expression in multiple human cancer tissue

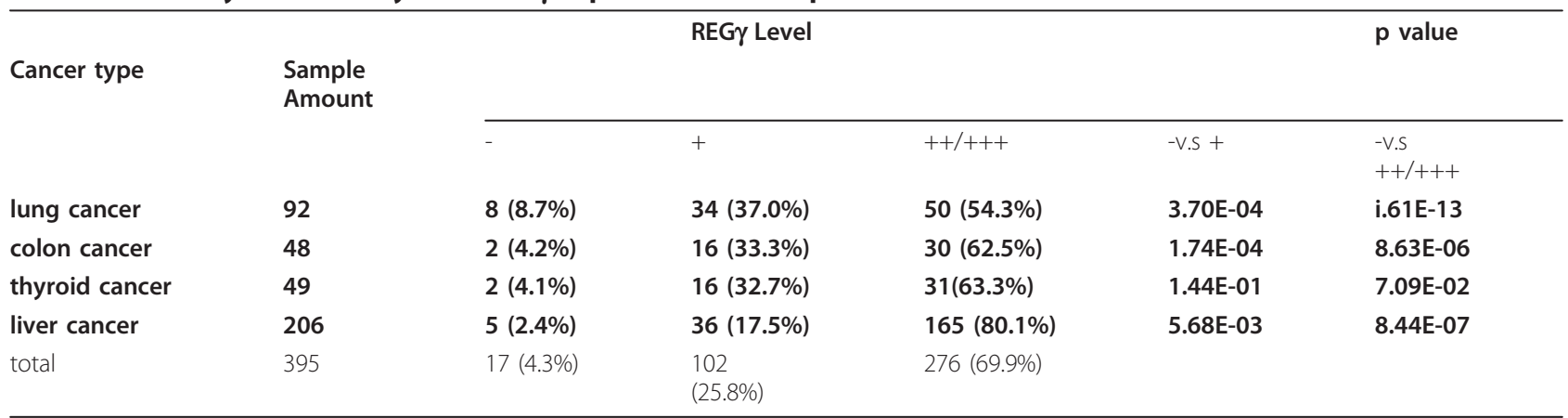

REG $\gamma$ expression status was scored according to description in Method \& Materials. Overexpression rate of REG $\gamma$ in each cancer was calculated based on the number of cases scored ++ and above. - v.s + represents two sample weighted student t-test for unequal variance between - group and + group. For - v.s $++/++$ + , it's between - group and $++/+++$ group 


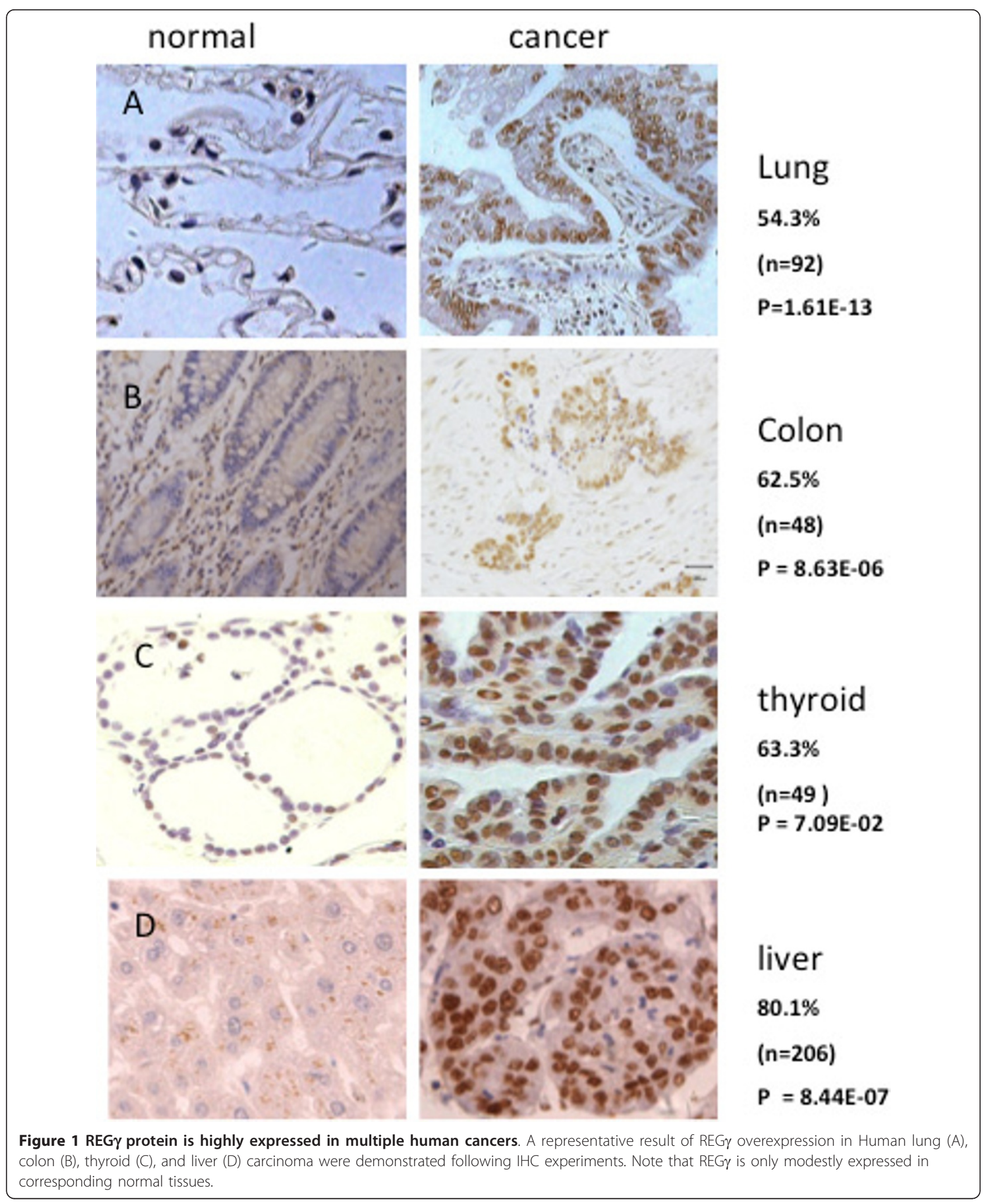




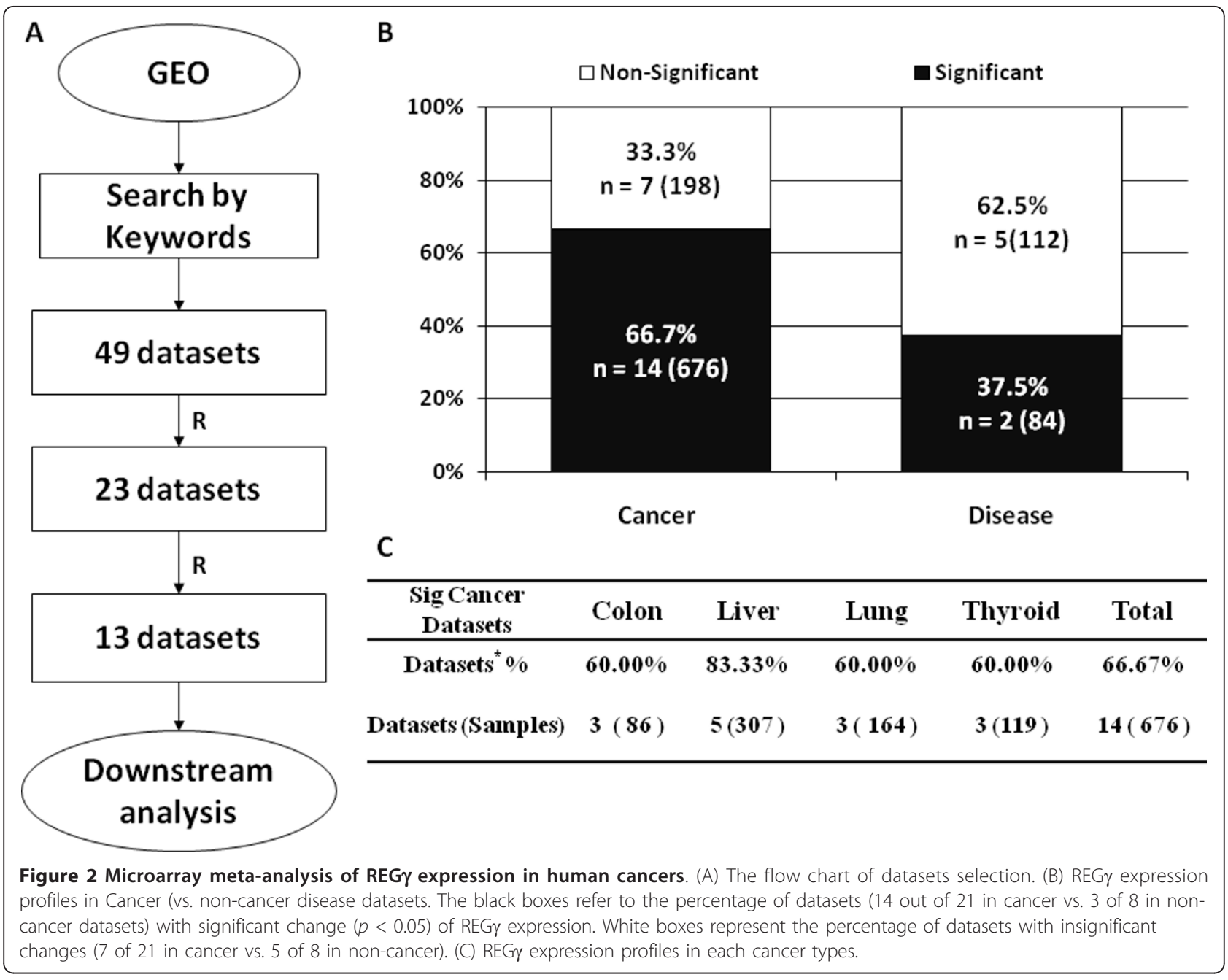

Correlation analysis links REG $\gamma$ to large numbers of cancer related genes/pathways

To explore potential mechanisms of REG $\gamma$ in cancer development, we further investigated genes whose expression is highly related to REG $\gamma$ expression in the four cancer types profiled. A statistical meta-analysis based on Pearson correlation coefficient (PCC) was conducted on the defined (REG $\gamma$ differential expressed) datasets (Additional file 4: Table S4). The correlation between REG $\gamma$ and every other gene in these datasets were calculated and evaluated statistically. With the assumption that a high absolute PCC value would reflect a potentially close relation to REG $\gamma$ functionally, only genes bearing high PCC scores were selected for subsequent studies.

To estimate that the approach we used in our analysis could indeed generate meaningful results, we first set up a PCC cutoff value $+/-0.6$ in at least one dataset for a pilot test. Since previous study [24,25] has demonstrated REG $\gamma$ mediated regulation of p53, we examined if p53 targets can be identified among REG $\gamma$ highly correlated genes. A total of 29 published genes in p53 signaling pathway were identified as significantly correlated with REG $\gamma$ (Additional file 5: Table S5), indicating that our normalized datasets contain valuable information required for further analysis.

By more stringent PCC-value based criteria, we screened genes highly-correlated with REG $\gamma$ and identified a total of 588 genes, with 521 positively correlated, 99 negatively correlated, and 31 being both negatively and positively correlated (Additional file 6: Table S6). Among these genes strongly correlated with REG $\gamma, 467$ were identified from colon cancer, while 75, 53, and 25 genes were from lung, thyroid and liver cancer respectively. Interestingly, multiple cancers shared significant amount of these genes. Based on all calculated results, there were $32 \%$ genes in two cancers, $43 \%$ genes in three cancers and $21 \%$ genes in four cancers simultaneously (Additional file 7: Figure S1A). The PCC between REG $\gamma$ and all other genes in each dataset are shown in Figure 4A. The range of the PCC 

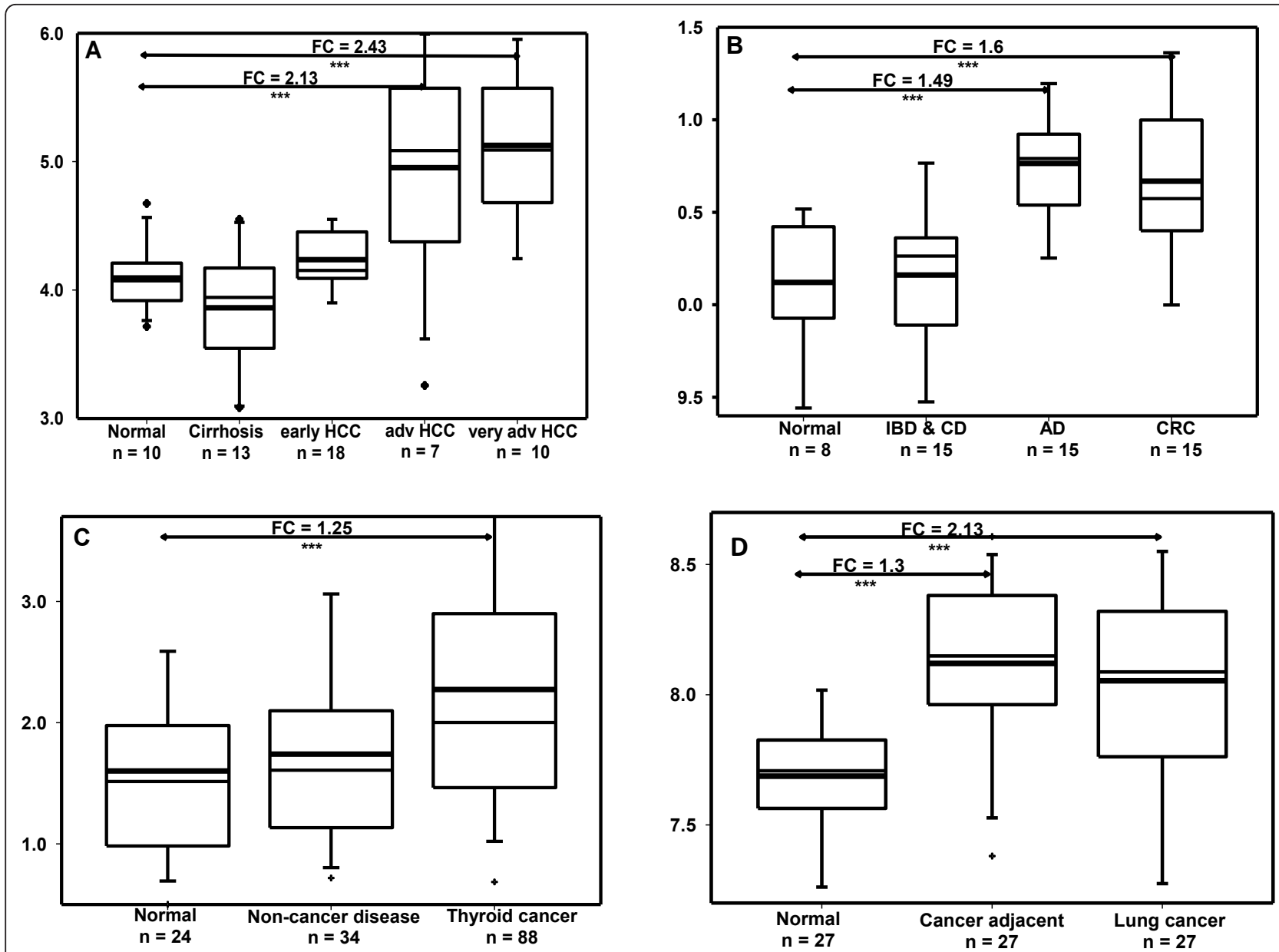

Figure 3 REG $\gamma$ expression values and variability in classified human cancers. Representative REG $\gamma$ expression fold-change values in pathologically classified, stage-specific cancers, non-cancer diseases and normal control datasets originated from liver (A), colon (B), thyroid (C) and lung (D) tissues were shown in box plot which signifies the upper and lower quartiles. The median is represented by a thin line and mean is represented by a bold line. The upper and lower limit refers to $95 \%$ and $5 \%$ data values. ${ }^{* * *}$ refers to $p<0.05,{ }^{*}$ denotes $p>0.05$. FC: fold change between two groups, HCC: hepatocelluar carcinoma, IBD: inflammatory bowel disease, CD: Crohn's disease, AD: Adenocarcinoma, CRC: Colorectal carcinoma.

plot reflects more positive-correlation points than negative ones, suggesting that more genes are positively correlated with REG $\gamma$ expression.

To understand functional diversities of the genes computationally correlated with REG $\gamma$, we performed Ingenuity pathway analysis of the 588 genes. Our analysis displayed that all mapped genes were functionally annotated into 500 pathways in which 207 were statistically significant $(P<0.01)$. Among the 207 pathways analyzed, 20 cancer pathways (10\%), 102 cancer related pathways (49\%), and 85 other pathways (41\%) were classified (Figure 4B). The top 15 pathways based on statistic significance (p-values) are shown in Additional file 7: S1B. Cancer related pathways were composed of 86 pathways related to cell cycle regulation, 9 pathways in apoptosis, and 7 pathways in cell growth (Additional file 6: Table S6). Due to the presence of subset of genes in multiple pathways, such as
Myc (in 37 function annotation pathways), HSP90AB1 (in 11 pathways), ILF2 and ODC1 (in 6 pathways respectively), the number of genes in cancer, cancer-related and other pathways were 163,168 , and 218 respectively with overlaps indicated in Figure 4C. Detailed information of pathway analysis is included in Additional file 6: Table S6. Based on Ingenuity analysis of cancers with REG $\gamma$ overexpression, our results indicate that over $50 \%$ of REG $\gamma$ highly-correlated genes/pathways are cancer or cancer-related.

We also validated our pathway analysis of REG $\gamma$-correlated genes by applying all 588 REG $\gamma$ highly correlated genes to KEGG pathway annotation. The results were consistent with Ingenuity analysis whereby cell cycle and cancer pathways were ranked among the top (Additional file 8: Table S7). Based on these annotation analyses, we discovered that REG $\gamma$ is linked to large numbers of cancer 


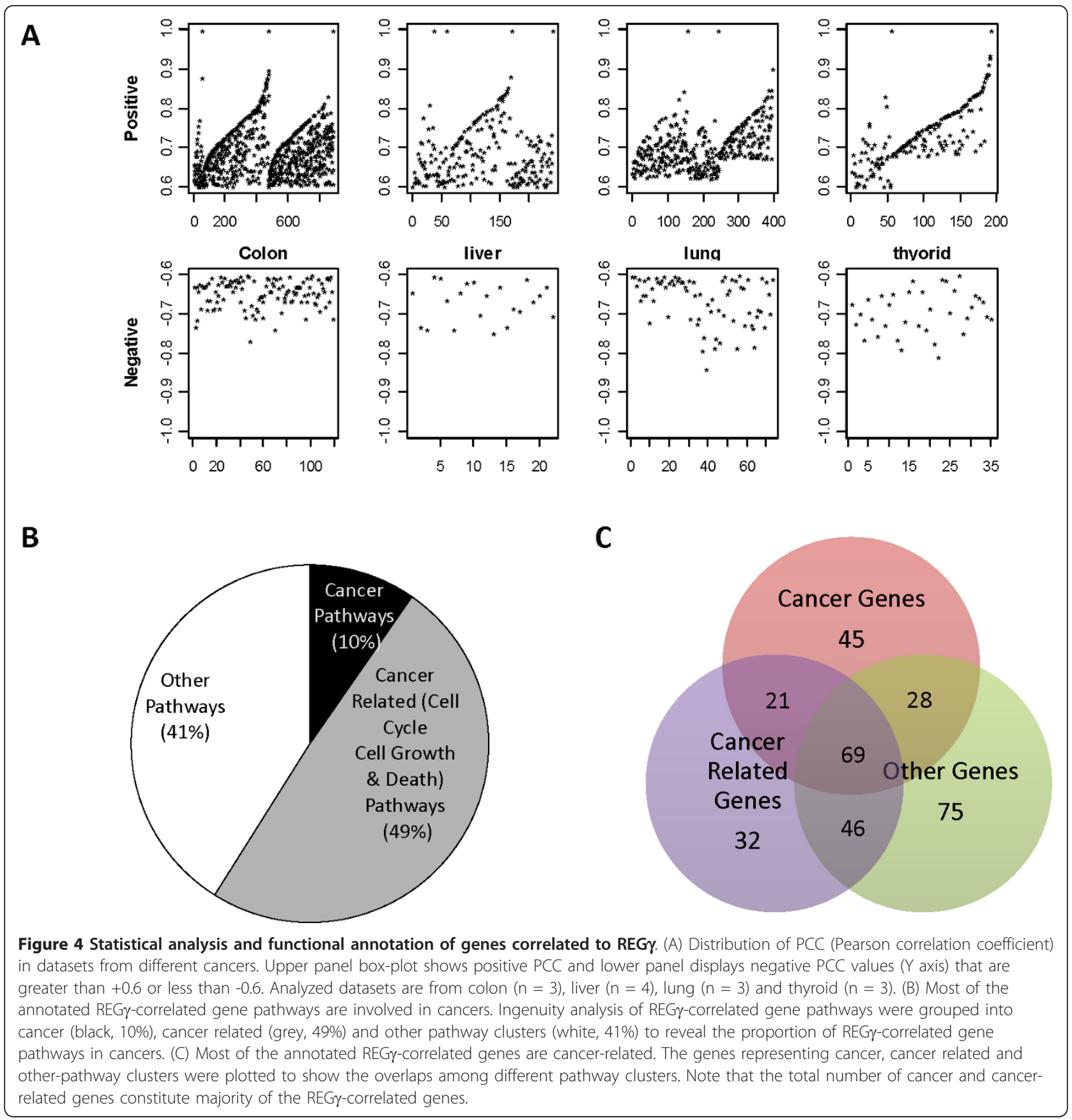

related genes, including Myc \& RAN in oncogenic pathway, BUB3 in spindle check-point function, BTG2 in cell cycle transition, DDB1 in DNA damage repair, DAPK2 in programmed cell death, in addition to genes in the p53 pathway like PTEN. We also observed that proteasome, ubiquitin-mediated proteolysis, and metabolic pathways were listed among the top of the 110 pathways covering 125 genes. Gene signaling pathways identified in KEGG analysis also include MAPK, Wnt, Jak-STAT, Neurotrophin, TGF- $\beta$, mTOR, and VEGF pathways. A battery of interesting genes were observed in the "other pathways" cluster, encompassing genes in spliceosome like HNRNPC \& SFRS3, genes in aminoacyl-tRNA biosynthesis such as DARS \& KARS, genes in immune response containing TNFSF10 \& MET, as well as genes involved in epigenetic regulation, including SUV39H1, H2, PRMT5, etc.

To illustrate potential links between the gene products among the REG $\gamma$ correlated genes, we conducted further analysis of protein-protein interaction (PPI) network using STRING [19], which is an online database of known and 
predicted protein interactions. This generated network (Additional file 9: Figure S2) integrated information from experimental repositories, computational prediction and published collections, and showed their interaction with default parameters. PPI network revealed potential interactions among five clusters of REG $\gamma$ correlated gene products, including those in metabolic pathways, proteasome pathways, cell cycle related pathways, DNA repair pathways, and tRNA biosynthesis pathways. These results provide additional information for future study of cellular function of REG $\gamma$ as well as its regulation.

\section{Confirmatory analysis of REG $\gamma$ correlated genes from bioinformatic analysis}

Our computational analysis indicated strong correlation of REG $\gamma$ to genes regulated by p53 and in cancer related pathways. To validate our bioinformatics-based predictions, we selected 30 genes for expression analysis using specific cancer cell lines. In addition to genes associated with p53 pathways, we selected two representative genes from each of the top cancer/cancer related pathways, metabolic pathways as well as those appeared in KEGG and Ingenuity network analysis (Table 2). We made use of stable cell lines constitutively expressing a control shRNA $(\mathrm{shN})$ or a REG $\gamma$ specific shRNA (shR). Three pairs of shRNA expressing cell lines were originated from lung, colon, and thyroid (A549, HCT116, and ARO, Additional file 10: Figure S3). The REG $\gamma$ knockdown in HepG2 liver cancer cell lines was generated by introducing synthetic siRNA against REG $\gamma$ [26]. The significant differences of REG $\gamma$ expression between control (shN) and REG $\gamma$ knockdown in each pair of the cell lines allowed us to examine

Table 2 A summary of confirmatory qRT-PCR analysis of selective genes

\begin{tabular}{|c|c|c|c|c|c|}
\hline Tissue & Gene Symbol & PCR value & p-value & Status & Gene Annotation \\
\hline Colon & BTG2 & 1.25 & $1.5 \mathrm{E}-02$ & Consistent & A member of the BTG/Tob family \\
\hline Lung & DAPK2 & 1.35 & $6.0 \mathrm{E}-12$ & Consistent & Death-associated protein kinase 1 (DAPK1) \\
\hline Lung & GADD45B & 1.63 & $2.6 \mathrm{E}-02$ & Consistent & Growth arrest and DNA-damage-inducible \\
\hline Lung & SATB1 & 2.82 & 4.3E-04 & Consistent & SATB homeobox 1 \\
\hline Thyroid & $\mathrm{ABCA} 1$ & 1.68 & $2.8 \mathrm{E}-02$ & Consistent & ATP-binding cassette, sub-family A \\
\hline Thyroid & B3GALT4 & 1.32 & $2.0 \mathrm{E}-03$ & Consistent & $\begin{array}{l}\text { UDP-Gal:betaGlcNAc beta 1,3- } \\
\text { galactosyltransferase }\end{array}$ \\
\hline Thyroid & PTEN & 1.29 & 4.3E-02 & Consistent & $\begin{array}{l}\text { Phosphatase and tensin homolog, tumor } \\
\text { suppressor }\end{array}$ \\
\hline Colon & ССТ3 & 0.62 & $2.9 \mathrm{E}-03$ & Consistent & Member of the chaperonin \\
\hline Colon & DKC1 & 0.60 & $8.3 \mathrm{E}-04$ & Consistent & Dyskeratosis congenita 1, dyskerin \\
\hline Colon & HSP90AB1 & 0.82 & $2.4 \mathrm{E}-02$ & Consistent & A member of heat shock \\
\hline Colon & MYC & 0.57 & $6.7 \mathrm{E}-03$ & Consistent & myelocytomatosis viral oncogene homolog \\
\hline Colon & ODC1 & 0.80 & $1.3 \mathrm{E}-02$ & Consistent & A p53 target negatively regulated. \\
\hline Colon & RRM2 & 0.66 & 4.0E-02 & Consistent & ribonucleotide reductase $\mathrm{M} 2$ \\
\hline Liver & DDB1 & 0.63 & $3.6 \mathrm{E}-02$ & Consistent & Damage-specific DNA binding protein 1 \\
\hline Liver & HN1 & 0.65 & $1.3 \mathrm{E}-03$ & Consistent & Hematological and neurological expressed1 \\
\hline Liver & ILF2 & 0.79 & $5.7 \mathrm{E}-04$ & Consistent & Interleukin enhancer binding factor 2 \\
\hline Liver & RAN & 0.75 & $1.3 \mathrm{E}-02$ & Consistent & ras-related nuclear protein \\
\hline Lung & BUB3 & 0.82 & $8.2 \mathrm{E}-03$ & Consistent & Budding uninhibited by benzimidazoles 3 homolog \\
\hline Lung & USP14 & 0.74 & 2.7E-03 & Consistent & Ubiquitin specific peptidase 14 \\
\hline \multirow[t]{2}{*}{ Thyroid } & ATR & 0.47 & $5.6 \mathrm{E}-03$ & Consistent & Ataxia telangiectasia and Rad3 related \\
\hline & & & Total & Consistent & $N=20(66.7 \%)$ \\
\hline Conlon & ACLY & 1.40 & 3.7E-05 & Inconsistent & ATP citrate lyase \\
\hline Thyroid & $\mathrm{TSC} 2$ & 2.18 & $1.4 \mathrm{E}-05$ & Inconsistent & Tuberous sclerosis 2, tumor suppressor \\
\hline Colon & CDK1 & 0.50 & $7.3 \mathrm{E}-07$ & Inconsistent & Cyclin-dependent kinase 1 \\
\hline Colon & SPPL2A & 0.38 & $3.5 \mathrm{E}-02$ & Inconsistent & Signal peptide peptidase-like $2 \mathrm{~A}$ \\
\hline Conlon & TUBG1 & 0.43 & $8.5 \mathrm{E}-05$ & Inconsistent & Tubulin, gamma 1 \\
\hline Liver & EHHADH & 0.61 & $4.4 \mathrm{E}-02$ & Inconsistent & Enoyl-CoA, hydratase/dehydrogenase \\
\hline Liver & CYP4F2 & 0.70 & $3.2 \mathrm{E}-02$ & Inconsistent & Cytochrome P450, family 4, subfamily F \\
\hline Lung & STARD8 & 0.66 & $7.5 \mathrm{E}-03$ & Inconsistent & A subfamily of Rho GTPase \\
\hline Lung & UNC13A & 4.73 & $8.2 \mathrm{E}-06$ & Inconsistent & Unc-13 homolog A \\
\hline \multirow[t]{2}{*}{ Thyroid } & PPAP2A & 0.63 & $2.8 \mathrm{E}-02$ & Inconsistent & Phosphatidic acid phosphatase type $2 \mathrm{~A}$ \\
\hline & & & Total & Inconsistent & $\mathrm{n}=10(33.3 \%)$ \\
\hline
\end{tabular}

PCR value was the expression level of qRT-PCR results in shR (REG $\gamma$ depletion) cells relative to that in shN (REG $\gamma$ expressing) cells (see Figure S4). The results were averaged from three independent experiments 
the relative levels of genes predicted to be highly correlated to REGy (Additional file 10: Figure S3). Real time RT-PCR was performed with specific primers for each gene in multiple pairs of cell lines or in a specific pair of cells dependent upon cancer-type specific bioinformatics data (Additional file 11: Table S8).

The relative expression ratios of each specific gene in shN and shR cancer cell lines were calculated from average of three independent experiments and the data with statistically significant changes $(p<0.05)$ between shN and shR cells were plotted (Figure $5 \mathrm{~A}$ and $5 \mathrm{~B}$ ). The expression of most genes $(66.7 \%, \mathrm{n}=20)$ in at least one cell line with or without REG $\gamma$ knockdown was consistent with predicted correlation to REG $\gamma$ levels (Table 2).

All genes validated by RT-PCR were applied into Ingenuity system for core analysis. Network information showing the link among these REG $\gamma$ correlated genes was displayed in Figure 6A. This analysis placed Myc as the hub of the interaction network and prompted us to perform further analysis on its biological significance. Interestingly, analysis of 11 colon cancer samples suggested significant positive correlation between cMyc and REG $\gamma$
(Figure 6B and 6C). The p53 target, PTEN, was also observed in this network analyses, reinforcing the close correlation among REG $\gamma$, p53 pathway, and other cancer related pathways.

\section{Discussion}

REG $\gamma$-proteasome system represents an emerging pathway recently recognized to be involved in cancer development. This study provides further links between REG $\gamma$ and multiple cancer related pathways by a combination of bioinfomatic analysis and molecular biological approach.

To our knowledge, this is the first computational study so far in REG $\gamma$ association with multiple cancers. We are also the first to demonstrate high expression of REG $\gamma$ in lung and liver cancers despite that overexpression of REG $\gamma$ in thyroid and colon cancer were reported [6,27]. Tissue array analyses of four different human cancers, including lung, colon, thyroid, and liver cancers, revealed significant increase of REG $\gamma$ protein in over $50 \%$ of these cancer samples. Bioinformatic analysis of human microarray gene expression profiles indicates that REG $\gamma$ gene expression is

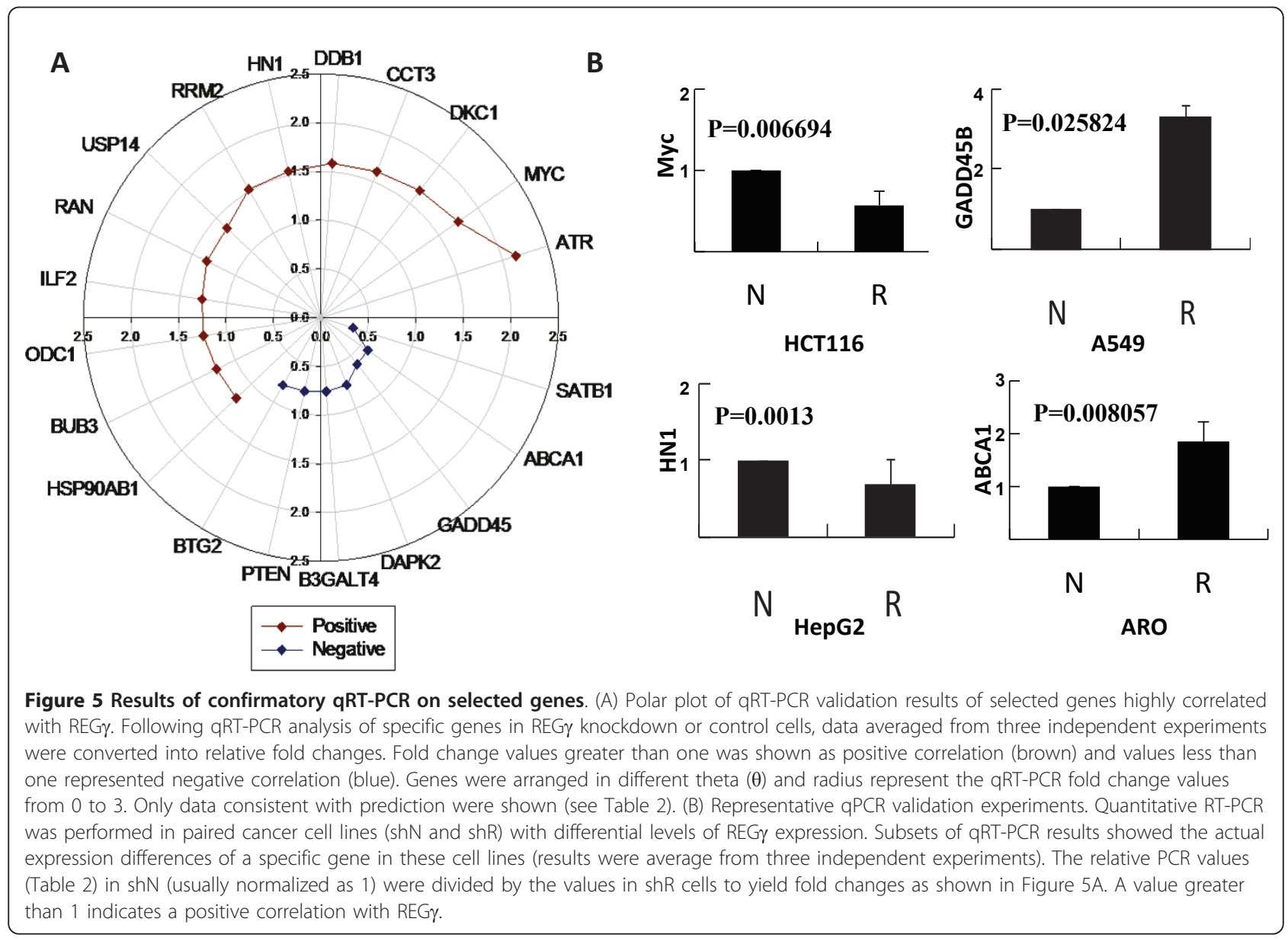




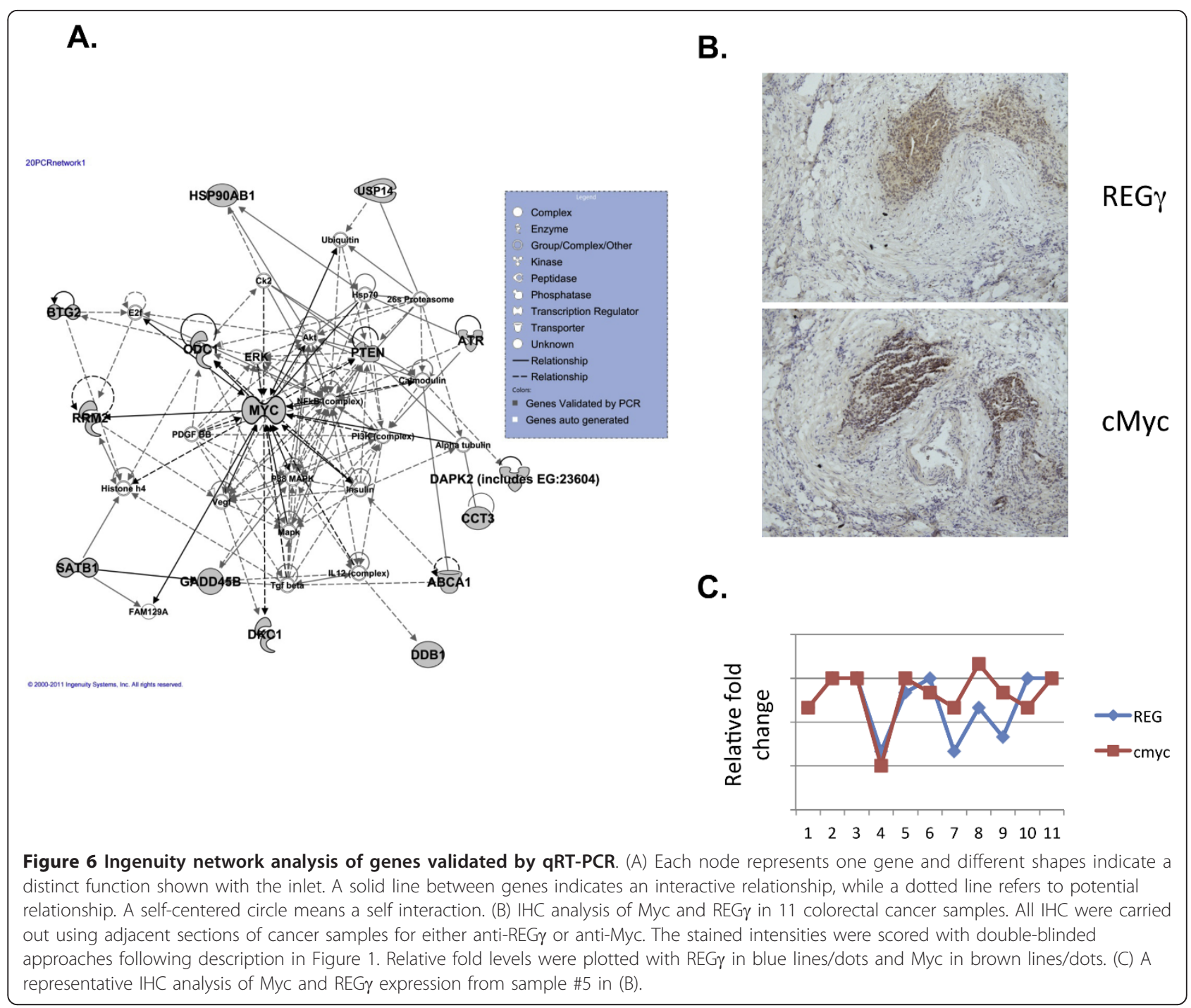

also increased in most of these human cancers, providing new evidence that REG $\gamma$-proteasome pathway may be involved in the development of multiple cancers.

Computational analysis of datasets from thyroid cancer with thyroid non-cancer disease and liver cancer with HCC clinical stage information indicated a potential correlation of gradual increase of REG $\gamma$ level with cancer stages (Figure 3A). Although the sample size and numbers are relatively small, the results suggest a potential of REG $\gamma$ as a prognostic cancer marker and hinted some molecular mechanisms linking REG $\gamma$ to development of cancers toward later stages or malignancy.

Our meta-analysis disclosed significant correlation between REG $\gamma$ and many genes in cancer and cancer related pathways from ingenuity analysis, including colorectal cancer, lung carcinoma, sarcoma, lymphoma, tumorigenesis, cell division and apoptosis related pathways etc. Importantly, genes downstream of the previously identified
REG $\gamma$ regulated proteins [24,25], p53, was found highly correlated with REG $\gamma$ expression. Despite that p53 mutation in different cancer may complicate the correlation status of its downstream target genes with REG $\gamma$, the overall high correlation values strongly support the previous finding that REG $\gamma$-mediated regulation of p53 may play an important role in cancer development. Annotation analysis indicated significant correlation of REG $\gamma$ with many different proteasome components, suggesting that REG $\gamma$ may be elevated and function together with other proteasome complexes. Recent studies have indicated important roles of the ubiquitin-mediated protein degradation pathway in cancer and application of proteasome inhibitors as a promising anti-cancer therapeutic approach [28]. An additional interesting finding is that numerous REG $\gamma$-correlated genes are involved in metabolism, particularly in energy metabolism (Additional file 8: Table S7). The link between cell metabolism and cancers has been well 
documented [29]. The result concurs a recent notion that cancer cell metabolisms are controlled by oncogenes and tumor suppressor genes [30].

The mathematical approaches of bioinformatics used in this study are quite standard [18]. To minimize the chance of acquiring false-positive results and ensure that most of the strong candidate genes are selected, we set rigorous criteria for all studies performed. The significance of our computational analysis has been underlined by laboratory validation experiments in 4 pairs of cancer cell lines in which differential expression of REG $\gamma$ was created in the same background to facilitate correlation studies. Results from quantitative analysis of selected genes were largely consistent with predicted correlations, suggesting powerful combination of bioinformatics and molecular biological studies in disclosing potentially novel functions of REG $\gamma$-proteasome in cancer progression. It is not unlikely that REG $\gamma$ could serve as a cancer marker, particularly for cancers with aggressive behavior.

Given that REG $\gamma$ mainly functions as a proteasome activator to induce protein degradation, the biological links between REG $\gamma$ and its correlated genes may reflect a result of direct or indirect regulation on transcription. Ingenuity analysis of validated gene network led our attention to the correlation between REG $\gamma$ and Myc gene. Previous research documented overexpression of both genes in colorectal cancers [31-33]. Coincident with the largest amount of datasets and highest REG $\gamma$ differential expression from colon cancers, the positive correlation between REG $\gamma$ and Myc was validated specifically in HTC116-shN and -shR cells. Since Myc functions as a transcription factor, we searched REG $\gamma$ promoter and found numerous Myc binding sites within $1.5 \mathrm{~KB}$ upstream REG $\gamma$ transcriptional initiation site (data not shown). Yet we could not exclude the possibility that REG $\gamma$ may target a negative regulator of Myc for degradation. Further experiments will be carried out to understand the molecular detail of these hypotheses. It is likely that elevated expression of Myc in certain cancer cells is one of the potential mechanisms contributing to higher expression of REG $\gamma$.

\section{Conclusions}

This study provides REG $\gamma$ expression profiles based on computational analysis of published microarray datasets and laboratory experiments on cancer samples. Data analysis links REG $\gamma$ to multiple cancer-related pathways. Our results indicate potentially important roles of REG $\gamma$ in multiple cancer types and implicate REG $\gamma$ as a putative cancer marker.

\section{Funding}

This work was supported by National Institutes of Health (1R01CA131914) and Norman Hackerman
Advanced Research Program (1082318401; PN0049490012-2009). This manuscript was also funded in part by the National Natural Science Foundation of China (30811120435, 30870503, 81071657); the Science and Technology Commission of Shanghai Municipality (06DZ22923, 11DZ2260300, 10JC1404200, 09ZZ41); the National Basic Research Program (2009CB918402, 2011CB504200); and the East China Normal University short-term oversee training program.

\section{Additional material}

Additional file 1: Table S2. A summary of 49 datasets collected for preliminary filtering. Cancer types were identified according to the sample origin disclosed. Accession numbers were acquired from GEO "Sample size" included all samples from cancer, disease and controls.

Additional file 2: Table S3. Datasets selected for analyzing genes differentially expressed A) All cancer datasets used for two sample tests. B) All non-cancer disease datasets used for two sample tests. C) Four datasets with staged information for two sample tests. (XLS 35 kb).

Additional file 3: Table S1. Detailed IHC information of REG $\gamma$ expression in multiple human cancers. REG $\gamma$ expression status was scored according to description in Materials \& Methods. Overexpression rate of REG $\gamma$ in each cancer was calculated based on the number of cases scored ++ and above. Pathological grading and histological information are provided for lung cancer $(A)$, colon cancer $(B)$, thyroid cancer (C) and liver cancer (D).

Additional file 4: Table S4. Information of 13 datasets for correlation analysis. The "Critical Value for test of PCC in two tails" is variable depending on sample size of each dataset. The "Top \% selected" was arbitrarily set.

Additional file 5: Table S5. Genes in p53 pathway following primary correlation analysis. The twenty nine p53 regulated genes were screened following a pilot, less stringent correlation criteria (PCC +/- 0.6; binomial coefficient as 1). Predicted correlation in datasets was shown.

Additional file 6: Table S6. Ingenuity pathway analysis of genes highly correlated with REG $\gamma$. The "Function Annotation" column shows the annotated pathways from Ingenuity knowledge base. "Molecules" refer to genes in each pathway. Pathways were ordered according to $\mathrm{p}$ values.

Additional file 7: Figure S1. Features of genes highly correlated to REG $\gamma$ following PCC and ingenuity analysis. (A) REG $\gamma$ highly-correlated genes shared in different cancers. The number in $X$ axis at the bottom of each column represents the number of cancer types sharing REG $\gamma$ highly-correlated genes. $Y$ axis refers to the percentage of REG $\gamma$ highlycorrelated gene shared in cancers. (B) Top 15 significant pathways in Ingenuity analysis of genes highly correlated with REG $\gamma$. The X-axis shows the bio-function annotation for each of the 15 pathways. The black bars (\# molecules) corresponding to Y-axis on the left represent the number of genes in each pathway. The crossed curve corresponding to Y-axis on the right shows the $\mathrm{p}$-value in logarithm based on 10. The straight line refers to a cutoff for significant $p$-value.

Additional file 8: Table S7 KEGG pathway analysis of genes highly correlated with REG $\gamma$. "KO" indicates the pathway map ID in the KEGG pathway database. "Gene Amount" represents the Gene number in each pathway form input genes.

Additional file 9: Figure S2 Protein-Protein interaction network of genes highly correlated with REG $\gamma$. Nodes of sphere stand for proteins. Colored lines represent different types of protein-protein relationships including: green for neighborhood, red for gene fusion, blue for cooccurrence, black for co-expression, purple for experiments validated, light blue for databases, yellow for text-mining, and gray lines for homology. Length of the lines stands for the score of functional-link. 
Additional file 10: Figure S3 RNA interference against REG $\gamma$ significantly attenuated REG $\gamma$ expression and function in different cancer cell lines. Different cancer cell lines were generated by stably integrating a control shRNA or an shRNA specifically targeting REG $\gamma$ (in A549, ARO and HCT116). RNA interference were also performed by transiently transfecting control (siN) and synthetic siRNA targeting REG $\gamma$ (siR) to HepG2 cells. Resulted cells normally expressing REG $\gamma$ (shN/siN) or with REG $\gamma$ depletion (shR/siR) showed expressional and functional differences as demonstrated by change of $\mathrm{p} 21$, the known REG $\gamma$ target.

Additional file 11: Table S8 Primers used in RT-PCR validation analysis. Sequences of gene-specific primer sets for RT-PCR analysis were displayed.

\section{Abbreviations}

GEO: Gene expression omnibus; IHC: Immunohistochemistry; HCC: Primary hepatocelluar carcinoma; PTC: Papillary thyroid carcinomas; ATC: Anaplastic thyroid carcinoma; IBD: Inflammatory bowel disease; CD: Crohn's disease; UC: Ulcerative colitis; PCC: Pearson's correlation coefficient.

\section{Author details}

${ }^{1}$ Institute of Biomedical Sciences, East China Normal University, 500 Dongchuan Rd., Shanghai 200241, China. ' ${ }^{2}$ Department of Colorectal Surgery, Xin-hua Hospital, Shanghai Jiao-tong Univerisy, Shanghai, People's Republic of China. ${ }^{3}$ Department of Pathology, the Second Chengdu Municipal Hospital, Chengdu, China. ${ }^{4}$ Department of Molecular and Cellular Biology, Baylor College of Medicine, One Baylor Plaza, Houston, TX 77030, USA.

\section{Authors' contributions}

$X L, S T, P e i-Z, J C, L C$, and $R C$ designed the study. JH, RC and ST performed bioinformatics and statistical data analyses. $X \mathrm{~L}, \mathrm{RC}$, and LC drafted the manuscript. YZ, GW and Ping-Z carried out quantitative PCR experiments and data interpretation. $Y Z, L$ and $Y Y$ performed IHC and Western blot analysis. All authors read and approved the final manuscript.

\section{Competing interests}

The authors declare that they have no competing interests.

Received: 22 July 2011 Accepted: 23 February 2012

Published: 23 February 2012

\section{References}

1. Mao I, Liu J, Li X, Luo H: REGgamma, a proteasome activator and beyond. Cellular Mol Life Sci 2008, 65:3971-3980.

2. Okamura T, Taniguchi S, Ohkura T, Yoshida A, Shimizu H, Hisatome I, Shiqemasa C: Abnormally high expression of proteasome activatorgamma in thyroid neoplasm. JCEM 2003, 88:1374-1383.

3. Markus R: Identification of PSME3 as a Novel Serum Tumor Marker for Colorectal Cancer by combing two-dimensional polyacrylamide gel electrophoresis with a strictly mass spectrometry-based approach for data analysis. Mol Cellular Proteomics 2006, 1:2092-2101.

4. Jia HL, Ye QH, Qin $L X$, Budhu A, Forgues $M$, Chen $Y$, Liu YK, Sun HC Wang $L$, Lu HZ, et al: Gene expression profiling reveals potential biomarkers of human hepatocellular carcinoma. Clin Cancer Res 2007, 13:1133-1139.

5. Jiang X, Tan J, Li J, Kivimae S, Yang X, Zhuang L, Lee PL, Chan MT, Stanton LW, Liu ET, et al: DACT3 is an epigenetic regulator of Wnt/betacatenin signaling in colorectal cancer and is a therapeutic target of histone modifications. Cancer Cell 2008, 13:529-541.

6. Hong Y, Ho KS, Eu KW, Cheah PY: A susceptibility gene set for early onset colorectal cancer that integrates diverse signaling pathways: implication for tumorigenesis. Clin Cancer Res 2007, 13:1107-1114.

7. Chandran UR, Ma C, Dhir R, Bisceglia M, Lyons-Weiler M, Liang W, Michalopoulos G, Becich M, Monzon FA: Gene expression profiles of prostate cancer reveal involvement of multiple molecular pathways in the metastatic process. BMC Cancer 2007, 7:64.

8. Ye QH, Qin LX, Forgues M, He P, Kim JW, Peng AC, Simon R, Li Y, Robles Al, Chen $Y$, et al: Predicting hepatitis B virus-positive metastatic hepatocellular carcinomas using gene expression profiling and supervised machine learning. Nat Med 2003, 9:416-423.

9. Okamura T, Taniguchi S, Ohkura T, Yoshida A, Shimizu H, Sakai M, Maeta H, Fukui H, Ueta Y, Hisatome I, Shigemasa C: Abnormally high expression of proteasome activator-gamma in thyroid neoplasm. J Clin Endocrinol Metab 2003, 88:1374-1383.

10. Barrett T, Edgar R: Gene expression omnibus: microarray data storage, submission, retrieval, and analysis. Methods Enzymol 2006, 411:352-369.

11. Shi L, Jones WD, Jensen RV, Harris SC, Perkins RG, Goodsaid FM, Guo L, Croner $L$, Boysen $C$, Fang $H$, et al: The balance of reproducibility, sensitivity, and specificity of lists of differentially expressed genes in microarray studies. BMC Bioinformatics 2008, 9(Suppl 9):S10.

12. Maouche S, Poirier O, Godefroy T, Olaso R, Gut I, Collet JP, Montalescot G, Cambien F: Performance comparison of two microarray platforms to assess differential gene expression in human monocyte and macrophage cells. BMC Genomics 2008, 9:302.

13. Sean D, Meltzer PS: GEOquery: a bridge between the Gene Expression Omnibus (GEO) and BioConductor. Bioinformatics 2007, 23:1846-1847.

14. Fox RJ, Dimmic MW: A two-sample Bayesian t-test for microarray data. BMC Bioinformatics 2006, 7:126.

15. Smyth GK: Linear models and empirical bayes methods for assessing differential expression in microarray experiments. Stat Appl Genet Mol Biol 2004, 3:Article3.

16. Baldi P, Long AD: A Bayesian framework for the analysis of microarray expression data: regularized $\mathrm{t}$-test and statistical inferences of gene changes. Bioinformatics 2001, 17:509-519.

17. Mansson R, Tsapogas P, Akerlund M, Lagergren A, Gisler R, Sigvardsson M: Pearson correlation analysis of microarray data allows for the identification of genetic targets for early B-cell factor. J Biol Chem 2004, 279:17905-17913.

18. Rosner B: Fundamentals of Biostatistics. 6 edition. Belmont, CA: ThomsonBrooks/Cole; 2006.

19. Jensen $L$, Kuhn M, Stark M, Chaffron S, Creevey C, Muller J, Doerks T, Julien P, Roth A, Simonovic M, et al: STRING 8-a global view on proteins and their functional interactions in 630 organisms. Nucleic Acids Res 2009, 37:D412-416.

20. Su L, Chang CW, Wu YC, Chen KC, Lin CJ, Liang SC, Lin CH, Whang-Peng J, Hsu SL, Chen CH, Huang CY: Selection of DDX5 as a novel internal control for Q-RT-PCR from microarray data using a block bootstrap resampling scheme. BMC Genomics 2007, 8:140.

21. Fontaine JF, Mirebeau-Prunier D, Franc B, Triau S, Rodien P, Houlgatte R, Malthiery $Y$, Savagner F: Microarray analysis refines classification of nonmedullary thyroid tumours of uncertain malignancy. Oncogene 2008, 27:2228-2236.

22. Gyorffy B, Molnar B, Lage H, Szallasi Z, Eklund AC: Evaluation of microarray preprocessing algorithms based on concordance with RT-PCR in clinical samples. PLoS One 2009, 4:e5645.

23. Wurmbach E, Chen YB, Khitrov G, Zhang W, Roayaie S, Schwartz M, Fiel I, Thung S, Mazzaferro V, Bruix J, et al: Genome-wide molecular profiles of HCV-induced dysplasia and hepatocellular carcinoma. Hepatology 2007, 45:938-947.

24. Liu J, Yu G, Zhao Y, Zhao D, Wang Y, Wang L, Li L, Zeng Y, Dang Y, Wang $C$, et al: REG\{gamma\} modulates $p 53$ activity by regulating its cellular localization. J Cell Sci 2010, 123:4076-4084.

25. Zhang Z, Zhang R: Proteasome activator PA28 gamma regulates $p 53$ by enhancing its MDM2-mediated degradation. EMBO J 2008, 27:852-864.

26. Li X, Lonard DM, Jung SY, Malovannaya A, Feng Q, Qin J, Tsai SY, Tsai MJ, O'Malley BW: The SRC-3/AIB1 coactivator is degraded in a ubiquitin- and ATP-independent manner by the REGgamma proteasome. Cell 2006, 124:381-392.

27. Salvatore G, Nappi TC, Salerno P, Jiang Y, Garbi C, Ugolini C, Miccoli P, Basolo F, Castellone MD, Cirafici AM, et al: A cell proliferation and chromosomal instability signature in anaplastic thyroid carcinoma. Cancer Res 2007, 67:10148-10158.

28. Levine AJ, Puzio-Kuter AM: The control of the metabolic switch in cancers by oncogenes and tumor suppressor genes. Science 2010, 330:1340-1344.

29. Burger AM, Seth AK: The ubiquitin-mediated protein degradation pathway in cancer: therapeutic implications. Eur I Cancer 2004, 40:2217-2229.

30. Wellen KE, Thompson CB: Cellular metabolic stress: considering how cells respond to nutrient excess. Mol Cell 2010, 40:323-332. 
31. Yu G, Zhao Y, He J, Lonard DM, Mao CA, Wang G, Li M, Li X: Comparative analysis of REG\{gamma\} expression in mouse and human tissues. J Mol Cell Biol 2010, 2:192-198.

32. Goh HS, Soong TW, Lee YS, Ho J, Goh CR: c-myc Oncogene expression in colorectal cancer: its use in prognosis and role in colorectal carcinogenesis. Ann Acad Med Singapore 1990, 19:139-141.

33. Sikora K, Chan S, Evan G, Gabra H, Markham N, Stewart J, Watson J: c-myc oncogene expression in colorectal cancer. Cancer 1987, 59:1289-1295.

Pre-publication history

The pre-publication history for this paper can be accessed here:

http://www.biomedcentral.com/1471-2407/12/75/prepub

doi:10.1186/1471-2407-12-75

Cite this article as: He et al:: REG $\gamma$ is associated with multiple oncogenic pathways in human cancers. BMC Cancer 2012 12:75.

\section{Submit your next manuscript to BioMed Central} and take full advantage of:

- Convenient online submission

- Thorough peer review

- No space constraints or color figure charges

- Immediate publication on acceptance

- Inclusion in PubMed, CAS, Scopus and Google Scholar

- Research which is freely available for redistribution

Submit your manuscript at www.biomedcentral.com/submit 